\title{
Suscetibilidade Comparativa a Herbicidas Pós-Emergentes de BiótIPOS DE Digitaria ciliaris RESISTENTE E SUSCETÍvel AOS INIBIDORES DA ACCASE ${ }^{1}$
}

\author{
Relative Susceptibility to Post-Emergence Herbicides of ACCase-Resistant and Susceptible \\ Biotypes of Digitaria ciliaris
}

\author{
LÓPEZ-OVEJERO, R.F. ${ }^{2}$, CARVALHO, S.J.P. ${ }^{3}$, NICOLAI, M. ${ }^{3}$ e CHRISTOFFOLETI, P.J. ${ }^{4}$
}

\begin{abstract}
RESUMO - Esta pesquisa foi conduzida com o objetivo de avaliar a possibilidade de resistência múltipla aos herbicidas inibidores da ACCase, ALS e síntese de carotenos em um biótipo de capim-colchão (Digitaria ciliaris) resistente aos inibidores da ACCase. O experimento foi conduzido em casa de vegetação, utilizando dois biótipos de capim-colchão: um resistente (R) e outro suscetível (S) aos herbicidas inibidores da ACCase. O delineamento experimental utilizado foi o de blocos ao acaso, com quatro repetições. Foram utilizados 11 herbicidas: fluazifop-p-butil, haloxyfop-r-methyl, propaquizafop, sethoxydim, tepraloxydim, clethodim, fenoxaprop + clethodim, imazethapyr, nicosulfuron, iodosulfuron + foramsulfuron e mesotrione. Para cada herbicida, os tratamentos resultaram da combinação fatorial entre os dois biótipos de capim-colchão e 10 doses $(0,0 \mathrm{D} ; 0,016 \mathrm{D}$; 0,03D; 0,06D; 0,25D; 0,5D; 1,0D; 2,0D; 4,0D; e $16,0 \mathrm{D})$, em que D é a dose recomendada para cada produto. Os herbicidas foram aplicados sobre as plantas de capim-colchão em estádio de desenvolvimento de 3-4 folhas. Avaliou-se a porcentagem de controle aos 28 dias após a aplicação (DAA). O biótipo R foi confirmado como resistente aos herbicidas inibidores da ACCase (grupos químicos dos ariloxifenoxipropionatos e cicloexanodionas) com níveis variáveis de resistência cruzada. O biótipo $\mathrm{R}$ foi controlado satisfatoriamente pela dose de campo de todos os herbicidas alternativos aplicados (sulfoniluréias, imidazolinonas e inibidores da síntese de caroteno), excluindo a hipótese de resistência múltipla nesse biótipo de capim-colchão.
\end{abstract}

Palavras-chave: resistência cruzada, resistência múltipla, controle químico.

\begin{abstract}
This research was conducted to evaluate the possibility of multiple resistances to ACCase, ALS and carotene synthesis inhibiting herbicides in one ACCase-resistant biotype of crab-grass (Digitaria ciliaris). The experiment was conducted in a greenhouse, using two crab-grass biotypes: one resistant (R) and another susceptible (S) to ACCase inhibiting herbicides. The experimental design adopted was randomized blocks, with four replicates. Eleven herbicides were used: fluazifop-p-butyl, haloxyfop-r-methyl, propaquizafop, sethoxydim, tepraloxydim, clethodim, fenoxaprop + clethodim, imazethapyr, nicosulfuron, iodosulfuron + foramsulfuron and mesotrione. For each herbicide, the treatments resulted from a factorial combination between two biotypes of crabgrass and 10 rates $(0.0 D ; 0.016 D ; 0.03 D ; 0.06 D ; 0.25 D ; 0.5 D ; 1.0 D ; 2.0 D$; 4.OD and 16.OD); where $D$ is the recommended rate for each product. The herbicides were applied over crabgrass plants at the 3-4 leaf development stages. Percent control was evaluated 28 days after application (DAA). Biotype $R$ was confirmed as resistant to ACCase inhibiting herbicides (chemical groups of ariloxifenoxipropionates and cyclohexanodiones) with variable levels of crossresistance. Also, biotype $R$ was satisfactorily controlled by the field rates of all the alternative herbicides applied (sulfonilureas, imidazolinones and inhibitors of carotene synthesis), excluding the hypothesis of multiple resistance in this crab-grass biotype.
\end{abstract}

Keywords: cross-resistance, multiple resistances, chemical control.

Recebido para publicação em 3.3.2006 e na forma revisada em 10.11.2006.

Parte da tese do primeiro autor apresentada à ESALQ para obtenção do título de Doutor em Agronomia.

2 Eng.-Agr. Dr., <rfloveje @ esalq.usp.br>. ${ }^{3}$ Pós-Graduandos em Fitotecnia na Escola Superior de Agricultura "Luiz de Queiroz" ESALQ/USP, <sjpcarvalho@yahoo.com.br>, <marcelon@esalq.usp.br>; ${ }^{4}$ Professor Associado do Departamento de Produção Vegetal - ESALQ/USP, <pjchrist@esalq.usp.br>, Caixa Postal 09, 13418-900 Piracicaba-SP. 


\section{INTRODUÇÃO}

Atualmente, o controle de poáceas na cultura da soja (Glycine max) tem sido realizado principalmente por meio dos herbicidas inibidores da ACCase, aplicados em condição de pós-emergência. No entanto, a aplicação repetitiva do mesmo herbicida ou de herbicidas com o mesmo mecanismo de ação, na mesma área, eleva a probabilidade de seleção de biótipos de plantas daninhas resistentes a herbicidas (Christoffoleti \& López-Ovejero, 2004).

A resistência de plantas daninhas a herbicidas é definida como a capacidade natural e herdável de determinados biótipos, dentro de uma população, de sobreviver e se reproduzir após a exposição a doses de herbicidas que seriam letais a indivíduos normais (suscetíveis) da mesma espécie (Christoffoleti \& LópezOvejero, 2004). A resistência de plantas daninhas aos herbicidas é um fenômeno natural que ocorre espontaneamente em suas populações, não sendo, portanto, o herbicida o agente causador, mas sim selecionador dos indivíduos resistentes que se encontram em baixa freqüência inicial (Christoffoleti et al., 1994).

Denomina-se por resistência cruzada os casos de resistência que envolvem diferentes herbicidas que têm o mesmo sítio e/ou mecanismo de ação. Geralmente a resistência cruzada é conseqüência de uma alteração no sítio de ação enzimático do herbicida. A resistência múltipla, por sua vez, envolve herbicidas não relacionados quimicamente entre si e que apresentam mecanismos de ação diferenciados. Geralmente esta resistência é decorrente de processos metabólicos (Christoffoleti \& LópezOvejero, 2004).

Diversos casos de resistência de plantas daninhas a herbicidas têm sido apontados em localidades produtoras de soja, sendo alguns deles já estudados na literatura. Contudo, populações de capim-colchão (Digitaria ciliaris) têm surgido como novas manifestações que envolvem os herbicidas inibidores da ACCase (López-Ovejero et al., 2005). Neste caso, diversas estratégias devem ser adotadas no manejo da resistência, com atuação tanto na prevenção ao surgimento como no manejo das situações em que já existam plantas daninhas resistentes. Há vários trabalhos na literatura que abordam esse assunto, com destaque para
Christoffoleti et al. (1994) e Powles \& Holtum (1994).

Para manejo e prevenção do problema em sistemas de produção, são necessárias estratégias integradas que envolvam métodos culturais, físicos e mecânicos, além do químico (Monqueiro et al., 2000). Segundo Gressel \& Segel (1990), o uso de herbicidas alternativos, que apresentam controle satisfatório sobre o biótipo resistente, é recomendado, pelo menos em curto prazo. Para Boerboom (1999), o aspecto mais importante na prevenção e manejo da resistência é a recomendação de práticas e sistemas de produção em que a pressão de seleção de biótipos resistentes a determinado herbicida seja reduzida.

No entanto, a opção por um mecanismo de ação diferente também pode constituir um risco de seleção de biótipos de plantas daninhas também resistentes ao herbicida alternativo, se este for aplicado como única opção de controle (Gould, 1995). Assim, esta pesquisa foi conduzida com o objetivo de avaliar a possibilidade de resistência múltipla aos herbicidas inibidores da ACCase, ALS e síntese de carotenos em um biótipo de capim-colchão (Digitaria ciliaris) resistente aos inibidores da ACCase.

\section{MATERIAL E MÉTODOS}

O experimento foi conduzido em casa de vegetação do Departamento de Produção Vegetal da ESALG/USP, no município de Piracicaba, Estado de São Paulo, entre janeiro e março de 2005. No trabalho, foram utilizados dois biótipos de capim-colchão, sendo um sabidamente resistente (R) aos inibidores da ACCase (López-Ovejero et al., 2005), coletado no município de Piraí do Sul-PR, e um suscetível (S), coletado em área sem histórico de aplicação de inibidores da ACCase (município de Ponta Grossa-PR).

As sementes, depois de colhidas, foram acondicionadas em sacos de papel e etiquetadas, para posterior transporte até o laboratório da ESALQ/USP, onde foi realizado trabalho de identificação sistemática das amostras, constatando-se que as populações coletadas eram realmente constituídas de indivíduos da espécie Digitaria ciliaris. O armazenamento foi feito em local seco, à temperatura ambiente, até a instalação do experimento. 
Inicialmente, as sementes dos dois biótipos foram colocadas para germinar em caixas plásticas, com capacidade para $2 \mathrm{~L}$, preenchidas com substrato comercial. Guando as plântulas emersas se apresentavam em estádio de uma folha definitiva, foram transplantadas para vasos plásticos com capacidade para $0,5 \mathrm{~L}$, também preenchidos com substrato comercial, devidamente adubado, na densidade de quatro plantas por vaso. Os vasos foram mantidos em casa de vegetação e irrigados diariamente. $\mathrm{O}$ delineamento experimental utilizado foi o de blocos ao acaso, com quatro repetições.

Os herbicidas aplicados foram: inibidores da ACCase do grupo dos ariloxifenoxipropionatos-APP (fluazifop-p-butil, haloxyfop-r e propaquizafop), das cicloexanodionas-CHD (sethoxydim, tepraloxydim e clethodim) e mistura de ambos (fenoxaprop + clethodim); inibidores da ALS, do grupo das imidazolinonas (imazethapyr), utilizados na cultura da soja; inibidores da ALS, do grupo das sulfoniluréias (nicosulfuron e iodosulfuron + foramsulfuron); e inibidores da biossíntese de caroteno (mesotrione), utilizados na cultura do milho (Tabela 1). Os herbicidas com recomendação para a cultura do milho foram utilizados neste trabalho, pois a sucessão soja-milho é muito comum no Estado do Paraná - Brasil.

Para cada herbicida, os tratamentos foram resultados da combinação fatorial entre os dois biótipos de capim-colchão (R e S) e 10 doses de herbicidas (0,0D; 0,016D; 0,03D; 0,06D; 0,25D; 0,5D; 1,0D; 2,0D; 4,0D; e 16,0D), em que $\mathrm{D}$ é a dose recomendada para cada produto. Na ocasião em que as plantas apresentavam, em média, de 3 a 4 folhas, realizou-se a aplicação dos tratamentos herbicidas. As pulverizações foram feitas em câmara de aplicação fechada, utilizando-se ponta do tipo leque (Teejet 80.03E), com jato calibrado na altura de $0,50 \mathrm{~m}$ da superfície do alvo e um volume relativo de calda da ordem de $200 \mathrm{~L} \mathrm{ha}^{-1}$. Após a aplicação dos herbicidas, os vasos foram transportados novamente para a casa de vegetação, sendo irrigados apenas no dia seguinte, garantindo assim a absorção foliar dos produtos.

Tabela 1 - Tratamentos herbicidas aplicados sobre os biótipos resistente (R) e suscetível (S) de capim-colchão, com múltiplos da dose recomendada (D) e seus equivalentes em $\mathrm{g} \mathrm{ha}^{-1}$, para cada produto. Piracicaba, 2005

\begin{tabular}{|c|c|c|c|c|c|c|c|c|c|c|}
\hline \multirow{2}{*}{ Tratamento } & \multicolumn{10}{|c|}{ Múltiplos de dose $\mathrm{D}^{*}$} \\
\hline & 0,0 & 0,016 & 0,03 & 0,06 & 0,25 & 0,5 & 1,0 & 2,0 & 4,0 & 16,0 \\
\hline Herbicida & \multicolumn{10}{|c|}{ Dose equivalente $\mathrm{em}^{\mathrm{g} \mathrm{ha}^{-1}}$} \\
\hline \multicolumn{11}{|c|}{ Inibidores da ACCase } \\
\hline \multicolumn{11}{|c|}{ Ariloxifenoxipropionatos (APP) } \\
\hline fluazifop-p-butil & 0,0 & 2,9 & 5,9 & 11,7 & 46,9 & 93,7 & 187,5 & 375,0 & 750,0 & 3000,0 \\
\hline haloxyfop-r $\mathrm{r}^{1 /}$ & 0,0 & 1,0 & 1,9 & 3,7 & 15,6 & 31,2 & 62,4 & 124,8 & 249,6 & 998,4 \\
\hline propaquizafop ${ }^{\underline{1}}$ & 0,0 & 4,8 & 9,0 & 18,0 & 75,0 & 150,0 & 300,0 & 600,0 & $1.200,0$ & $4.800,0$ \\
\hline \multicolumn{11}{|c|}{ Cicloexanodionas (CHD) } \\
\hline sethoxydim$^{-1}$ & 0,0 & 3,6 & 7,2 & 13,8 & 57,5 & 115,0 & 230,0 & 460,0 & 920,0 & $3.680,0$ \\
\hline tepraloxydim ${ }^{-1}$ & 0,0 & 1,6 & 3,1 & 6,2 & 25,0 & 50,0 & 100,0 & 200,0 & 400,0 & $1.600,0$ \\
\hline clethodim $^{1}$ & 0,0 & 1,7 & 3,2 & 6,5 & 27,0 & 54,0 & 108,0 & 216,0 & 432,0 & $1.728,0$ \\
\hline \multicolumn{11}{|c|}{$\mathrm{APP}+\mathrm{CHD}$} \\
\hline $\begin{array}{l}\text { fenoxaprop }+ \\
\text { clethodim }^{1 /}\end{array}$ & 0,0 & $0,8+0,8$ & $1,5+1,5$ & $3+3$ & $12,5+12,5$ & $25+25$ & $50+50$ & $100+100$ & $200+200$ & $800+800$ \\
\hline \multicolumn{11}{|c|}{ Inibidores da ALS } \\
\hline imazethapyr $^{1 /}$ & 0,0 & 0,8 & 1,5 & 3,0 & 12,5 & 25,0 & 50,0 & 100,0 & 200,0 & 800,0 \\
\hline nicosulfuron $^{-1}$ & 0,0 & 0,8 & 1,5 & 3,0 & 12,5 & 25,0 & 50,0 & 100,0 & 200,0 & 800,0 \\
\hline $\begin{array}{l}\text { iodosulfuron }+ \\
\text { foramsulfuron }^{\underline{1}}\end{array}$ & 0,0 & $0,57+0,04$ & $1,1+0,07$ & $2,1+0,14$ & $9+0,6$ & $18+1,2$ & $36+2,4$ & $72+4,8$ & $144+9,6$ & $576+38,4$ \\
\hline \multicolumn{11}{|c|}{ Inibidor da síntese de carotenóides } \\
\hline mesotrione $^{1 /}$ & 0,0 & 2,3 & 4,3 & 8,6 & 36,0 & 72,0 & 144,0 & 288,0 & 576,0 & $2.304,0$ \\
\hline
\end{tabular}

* D é a dose recomendada para cada herbicida; ${ }^{\prime}$ À calda de pulverização foi adicionado óleo mineral ou adjuvante na concentração de $0,5 \%(\mathrm{v} / \mathrm{v})$. 
Foram realizadas avaliações de eficácia de controle, em dados percentuais, aos 28 dias após aplicação (DAA). Para eficácia de controle, foi atribuído $0 \%$ no caso da ausência de sintomas causados pelo herbicida e 100\% quando todas as plantas do vaso estavam mortas. Os dados foram inicialmente submetidos à aplicação do teste $\mathrm{F}$ sobre a análise de variância e, em seguida, ajustados ao modelo de regressão não-linear do tipo log-logístico proposto por Seefeld et al. (1995).

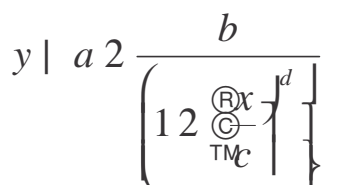

em que $y$ : porcentagem de controle; $x$. coeficiente da dose D; e $a, b, c$ e $d$ : parâmetros da curva, de modo que $a$ é o limite inferior da curva, $b$ é a diferença entre o ponto máximo e o mínimo da curva, $c$ é o coeficiente da dose $\mathrm{D}$ que proporciona $50 \%$ de resposta da variável e $d$ é a declividade da curva.

O modelo log-logístico apresenta vantagens, uma vez que um dos termos integrantes da equação (c) é uma estimativa do valor de $\mathrm{C}_{50}$ ou de $\mathrm{GR}_{50}$ (Christoffoleti, 2002). $\mathrm{O} \mathrm{C}_{50}$ (control by $50 \%$ ) e o $\mathrm{GR}_{50}$ (growth reduction by 50\%) são as doses do herbicida que proporcionam 50\% de controle ou de redução de massa da planta daninha, respectivamente (Christoffoleti \& López-Ovejero, 2004; Christoffoleti, 2002). Embora um dos parâmetros do modelo logístico (c) seja uma estimativa do valor de $\mathrm{C}_{50}$, optou-se por realizar seu cálculo matemático por meio da equação inversa, conforme discussão proposta por Carvalho et al. (2005).

Com os valores de $\mathrm{C}_{50}$, obteve-se o fator de resistência $(\mathrm{F})$ para cada combinação dos dois biótipos com os herbicidas testados. $\mathrm{O}$ fator de resistência $\left(\mathrm{F}=\mathrm{C}_{50}\right.$ resistente $/ \mathrm{C}_{50}$ suscetivel) expressa o número de vezes em que a dose necessária para controlar 50\% da população resistente é superior à dose que controla 50\% da população suscetível (Christoffoleti, 2002; Hall et al., 1998).

\section{RESULTADOS E DISCUSSÃO}

Os parâmetros $a, b, c$ e $d$ do modelo loglogístico e o coeficiente de determinação $\left(\mathrm{R}^{2}\right)$ para cada combinação entre biótipos e herbicidas são apresentados na Tabela 2. Para os dois grupos de herbicidas inibidores da ACCase estudados (APP ou CHD), o controle do biótipo S foi maior que os valores obtidos para o biótipo R. Quando foram utilizadas doses iguais ou superiores a $0,5 \mathrm{D}$, todos os herbicidas atingiram controles de $100 \%$ sobre o biótipo $\mathrm{S}$, aos 28 DAA; nas doses menores, a eficácia dos herbicidas utilizados foi diferenciada (Figuras 1 a 7).

No que se refere ao biótipo $\mathrm{R}$, os herbicidas fluazifop-p-butil, sethoxydim, clethodim e fenoxaprop + clethodim, quando aplicados na dose de 1D, obtiveram controles de aproximadamente $20,0,0,0,37,0$ e 50,0\%, respectivamente. Para os herbicidas haloxyfop-r, propaquizafop e tepraloxydim, aos 28 DAA, o controle obtido pela dose de 1D foi de $100 \%$ (Figuras 1 a 7). Nesse caso, para a aplicação a campo da dose $1 \mathrm{D}$ destes três últimos produtos sobre o biótipo R, não seriam observadas falhas de controle. Estes três herbicidas poderiam ser recomendados como alternativas de manejo do biótipo $\mathrm{R}$ nas doses registradas do produto, porém é importante ressaltar que niveis de resistência foram detectados também para esses produtos, conforme os valores de $\mathrm{C}_{50}$ discutidos a seguir (Tabela 3).

Com os valores de $\mathrm{C}_{50}$ obtidos, pode-se calcular o fator de resistência $(\mathrm{F})$ para o biótipo resistente quando submetido a todos os herbicidas estudados (Tabela 3). Para os herbicidas fluazifop-p-butil, haloxyfop-r e propaquizafop, pertencentes aos APP, foi necessária a aplicação de 45,963, 4,628 e 4,884 vezes mais produto sobre o biótipo R, quando comparado com o biótipo S, para obter o mesmo controle de $50 \%$, respectivamente. No caso dos herbicidas sethoxydim, tepraloxydim e clethodim, pertencentes aos CHD, foram necessários incrementos de 30,854, 2,334 e 8,680 vezes, respectivamente. Em se tratando da mistura de fenoxaprop + clethodim, foi necessária uma dose cerca de 9,149 vezes maior para o biótipo $R$ para obtenção do controle de 50\%, quando comparado com o biótipo suscetivel (Tabela 3).

A partir da análise do fator de resistência, observa-se que o biótipo resistente estudado apresentou padrões diferenciados de resistência cruzada aos herbicidas inibidores da ACCase. Os herbicidas fluazifop-p-butil, sethoxydim, clethodim e fenoxaprop + clethodim 
apresentaram maiores taxas de resistência $(>8,0)$ quando comparados aos herbicidas haloxyfopr, propaquizafop e tepraloxydim $(<4,9)$ (Tabela 3). Gazziero et al. (2000) e Christoffoleti et al.
(2001) relataram casos similares de espécies de plantas daninhas com diferentes niveis de resistência cruzada aos inibidores da ACCase no Brasil.

Tabela 2 - Parâmetros do modelo log-logístico ${ }^{1 /}$ e coeficiente de determinação $\left(\mathrm{R}^{2}\right)$ obtidos para os biótipos resistente (R) e suscetível (S) de capim-colchão. Piracicaba, 2005

\begin{tabular}{|l|c|c|c|c|c|c|}
\hline \multirow{2}{*}{ Herbicida } & \multirow{2}{*}{ Biótipo } & \multicolumn{4}{|c|}{ Parâmetro } \\
\cline { 3 - 7 } & & $\mathrm{a}$ & $\mathrm{b}$ & $\mathrm{c}$ & $\mathrm{d}$ & $\mathrm{R}^{2}$ \\
\hline \multirow{2}{*}{ Fluazifop-p-butil } & $\mathrm{R}$ & $-0,168$ & 102,688 & 3,099 & $-1,373$ & 0,992 \\
\hline \multirow{2}{*}{ Haloxyfop-r } & $\mathrm{S}$ & $-0,181$ & 100,233 & 0,063 & $-1,922$ & 0,999 \\
\hline \multirow{2}{*}{ Propaquizafop } & $\mathrm{R}$ & 0,001 & 100,000 & 0,250 & $-17,041$ & 0,999 \\
& $\mathrm{~S}$ & $-0,242$ & 100,114 & 0,051 & $-7,676$ & 0,999 \\
\hline \multirow{2}{*}{ Sethoxydim } & $\mathrm{R}$ & 0,001 & 100,000 & 0,276 & $-22,497$ & 0,999 \\
\hline \multirow{2}{*}{ Tepraloxydim } & $\mathrm{S}$ & $-1,522$ & 101,506 & 0,052 & $-2,713$ & 0,999 \\
\hline \multirow{2}{*}{ Clethodim } & $\mathrm{R}$ & 0,001 & 100,000 & 2,833 & $-35,144$ & 0,999 \\
\hline \multirow{2}{*}{ Fenoxaprop + clethodim } & $\mathrm{S}$ & 0,001 & 100,045 & 0,092 & $-3,589$ & 0,999 \\
\hline \multirow{2}{*}{ Imazethapyr } & $\mathrm{R}$ & 3,323 & 96,770 & 0,583 & $-10,218$ & 0,996 \\
\hline \multirow{2}{*}{ Nicosulfuron } & $\mathrm{S}$ & 0,001 & 100,000 & 0,250 & $-17,041$ & 0,999 \\
\hline \multirow{2}{*}{ Iodosulfuron + foramsulfuron } & $\mathrm{R}$ & 4,769 & 96,040 & 1,116 & $-6,194$ & 0,994 \\
\hline \multirow{2}{*}{ Mesotrione } & $\mathrm{S}$ & 0,002 & 100,144 & 0,063 & $-2,367$ & 0,999 \\
\hline & $\mathrm{R}$ & 1,620 & 100,937 & 0,997 & $-2,226$ & 0,997 \\
\hline & $\mathrm{S}$ & $-2,706$ & 102,646 & 0,081 & $-2,084$ & 0,997 \\
\hline & $\mathrm{R}$ & 0,360 & 104,279 & 0,286 & $-1,323$ & 0,969 \\
\cline { 2 - 7 } & $\mathrm{S}$ & 1,014 & 102,023 & 0,309 & $-1,699$ & 0,988 \\
\hline & $\mathrm{R}$ & 0,276 & 100,603 & 0,412 & $-3,658$ & 0,999 \\
\hline
\end{tabular}

${ }_{1}^{1 / \text { Modelo: }} y=a+\left(b /\left(1+(x / c)^{d}\right)\right)$.

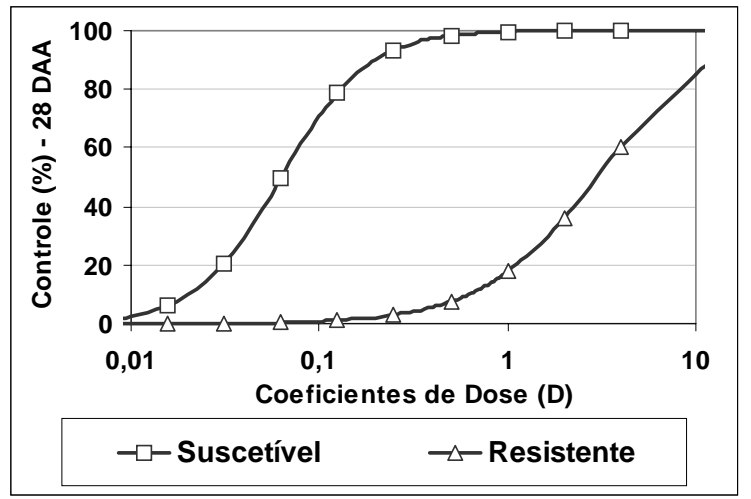

Figura 1 - Controle percentual de dois biótipos de capimcolchão, avaliado aos 28 DAA, quando submetidos à aplicação de fluazifop-p-butil. Piracicaba, 2005.

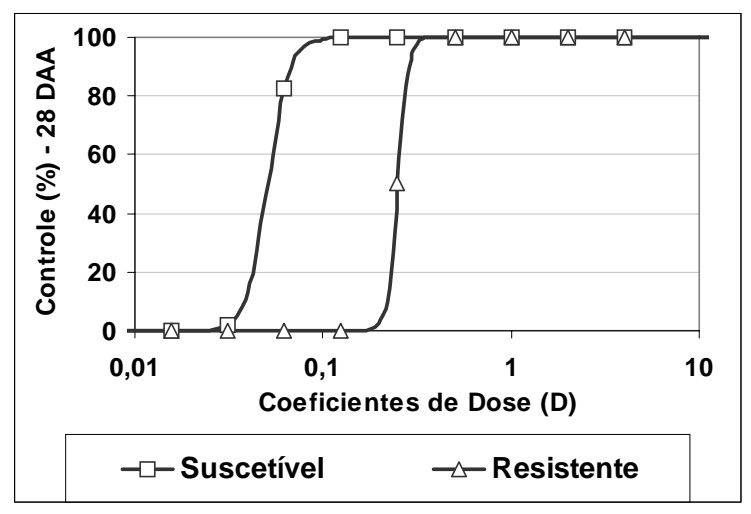

Figura 2 - Controle percentual de dois biótipos de capimcolchão, avaliado aos 28 DAA, quando submetidos à aplicação de haloxyfop-r. Piracicaba, 2005.

Planta Daninha, Viçosa-MG, v. 24, n. 4, p. 789-796, 2006 


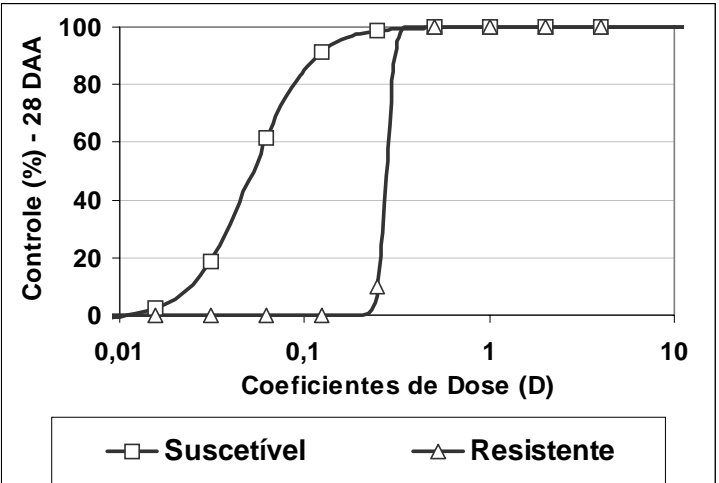

Figura 3 - Controle percentual de dois biótipos de capimcolchão, avaliado aos 28 DAA, quando submetidos à aplicação de propaquizafop. Piracicaba, 2005.

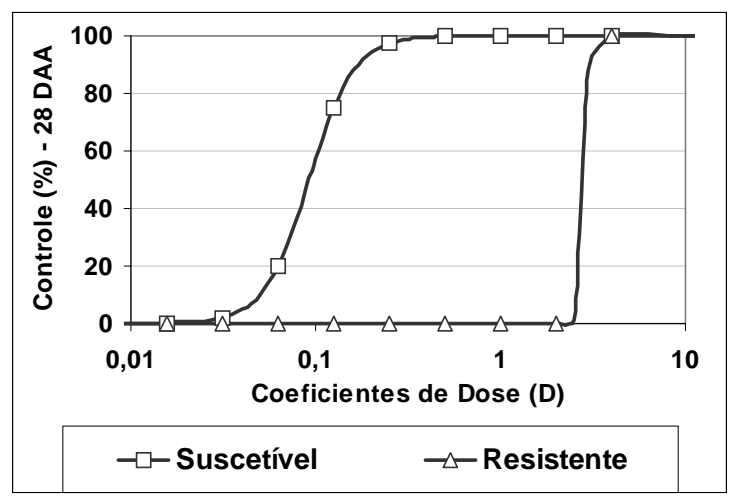

Figura 4 - Controle percentual de dois biótipos de capimcolchão, avaliado aos 28 DAA, quando submetidos à aplicação de sethoxydim. Piracicaba, 2005.

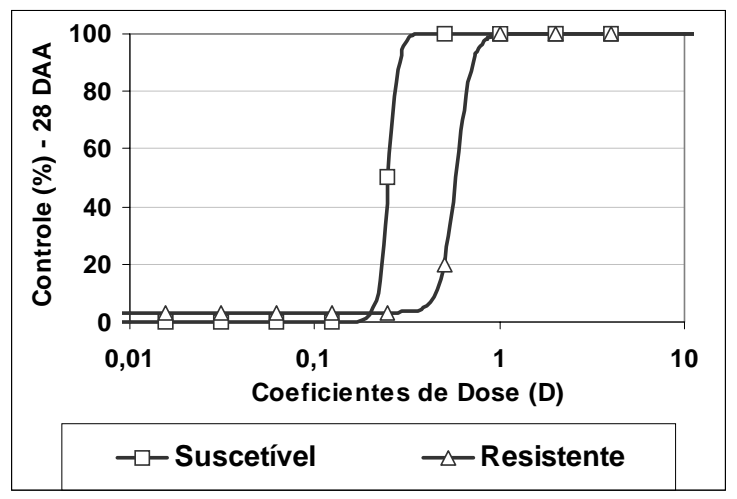

Figura 5 - Controle percentual de dois biótipos de capimcolchão, avaliado aos 28 DAA, quando submetidos à aplicação de tepraloxydim. Piracicaba, 2005.

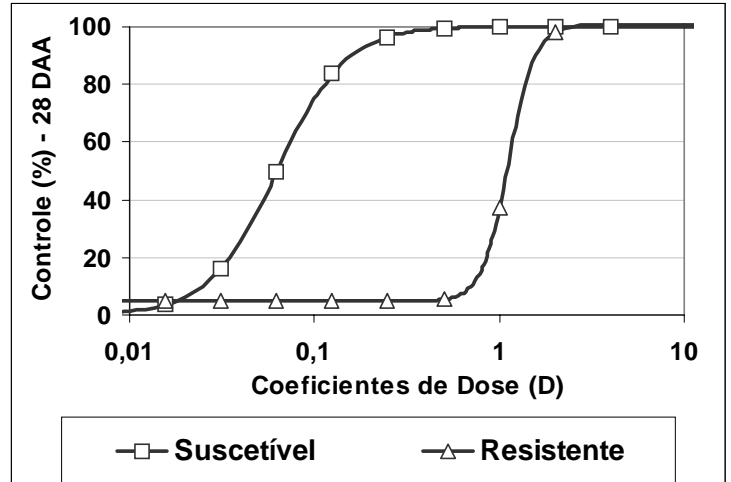

Figura 6 - Controle percentual de dois biótipos de capimcolchão, avaliado aos 28 DAA, quando submetidos à aplicação de clethodim. Piracicaba, 2005.

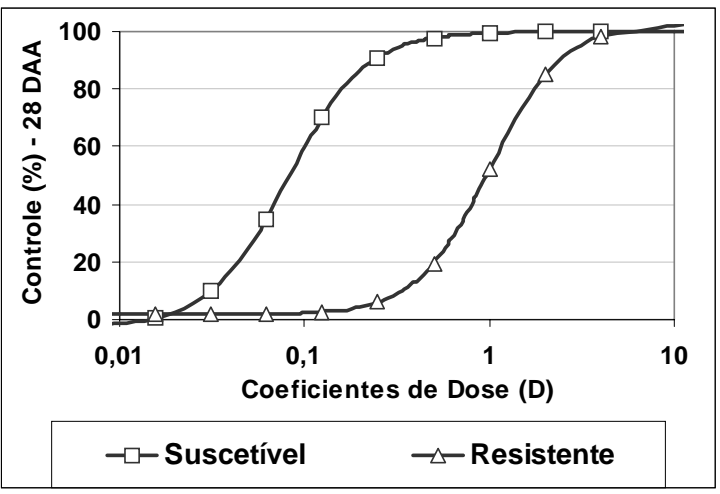

Figura 7 - Controle percentual de dois biótipos de capimcolchão, avaliado aos 28 DAA, quando submetidos à aplicação de fenoxaprop + clethodim. Piracicaba, 2005.

Tabela 3 - Valores de $\mathrm{C}_{50}$ obtidos através da aplicação da equação inversa do modelo logístico e fator de resistência $(\mathrm{F}=\mathrm{R} / \mathrm{S})$ para os biótipos resistente (R) e suscetível (S), de campim-colchão, aos 28 DAA. Piracicaba, 2005

\begin{tabular}{|c|c|c|c|}
\hline \multirow{2}{*}{ Herbicida } & \multicolumn{2}{|c|}{$\mathrm{C}_{50}{ }^{1 /}$} & \multirow{2}{*}{$\mathrm{F}$} \\
\hline & $\mathrm{S}$ & $\mathrm{R}$ & \\
\hline fluazifop-p-butil & 0,063 & 2,910 & 45,963 \\
\hline haloxyfop-r & 0,054 & 0,250 & 4,628 \\
\hline propaquizafop & 0,056 & 0,276 & 4,884 \\
\hline sethoxydim & 0,092 & 2,829 & 30,854 \\
\hline tepraloxydim & 0,250 & 0,583 & 2,334 \\
\hline clethodim & 0,063 & 0,543 & 8,680 \\
\hline fenoxaprop + clethodim & 0,091 & 0,829 & 9,149 \\
\hline imazethapyr & 0,270 & 0,252 & 0,933 \\
\hline nicosulfuron & 0,369 & 0,379 & 1,026 \\
\hline iodosulfuron + foramsulfuron & 0,300 & 0,308 & 1,025 \\
\hline mesotrione & 0,220 & 0,242 & 1,102 \\
\hline
\end{tabular}

${ }^{1 /}$ Coeficiente de dose $-\mathrm{D}$. 
Embora existam vários mecanismos de resistência associados aos herbicidas inibidores da ACCase, a maioria dos casos estudados demonstra que a resistência é conseqüência da insensibilidade enzimática (De Prado et al., 2004; Volemberg \& Stoltenberg, 2002). Aparentemente, os diferentes níveis de resistência cruzada apresentados pelos biótipos podem ser explicados pela existência de diferentes padrões da mutação enzimática (Volemberg \& Stoltenberg, 2002; Carvalho, 2004) e, também, pelas características de eficácia intrínsecas a cada molécula. Ainda, supõe-se que a mutação selecionada em determinada região esteja diretamente relacionada com os produtos mais aplicados na área. Essa suposição é verdadeira para o biótipo R, uma vez que os produtos mais aplicados nas lavouras do Paraná (fluazifop-p-butil e sethoxydim) foram aqueles que apresentaram os maiores niveis de resistência.

Todos os herbicidas inibidores da ALS e síntese de carotenos, quando aplicados na dose de 1D, apresentaram 100\% de controle sobre os biótipos R e S (Figuras 8 a 11). Os valores de $\mathrm{C}_{50}$ e $\mathrm{F}$ também foram calculados para os herbicidas imazethapyr, nicosulfuron, iodosulfuron + foramsulfuron e mesotrione, relacionados com os biótipos R e S (Tabela 3). Para todos esses herbicidas, o fator de resistência $(\mathrm{R} / \mathrm{S})$ foi de aproximadamente 1 , o que indica a inexistência de resistência múltipla no biótipo de capim-colchão estudado e, também, redução da probabilidade de o mecanismo de resistência ser do tipo metabólico.

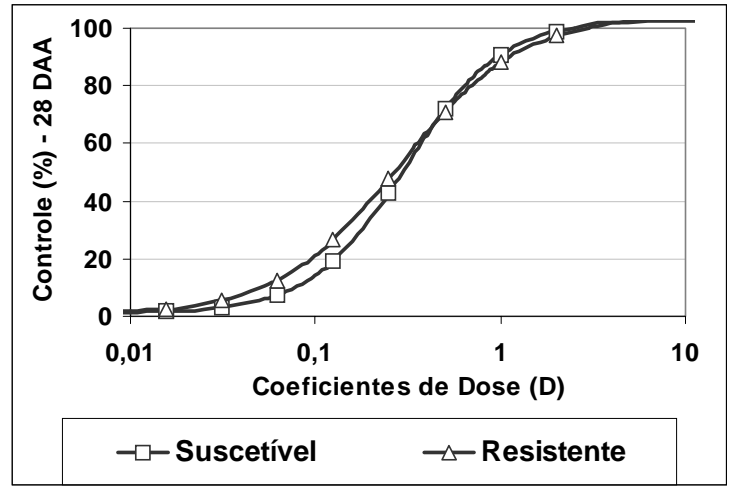

Figura 8 - Controle percentual de dois biótipos de capimcolchão, avaliado aos 28 DAA, quando submetidos à aplicação de imazethapyr. Piracicaba, 2005.

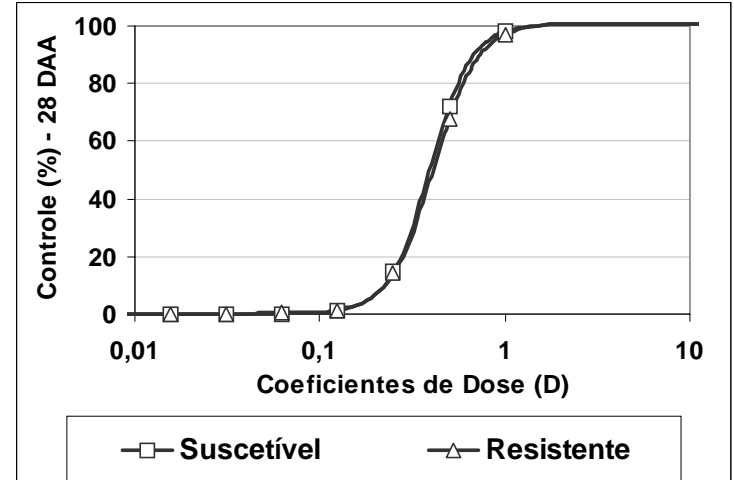

Figura 9 - Controle percentual de dois biótipos de capimcolchão, avaliado aos 28 DAA, quando submetidos à aplicação de nicosulfuron. Piracicaba, 2005.

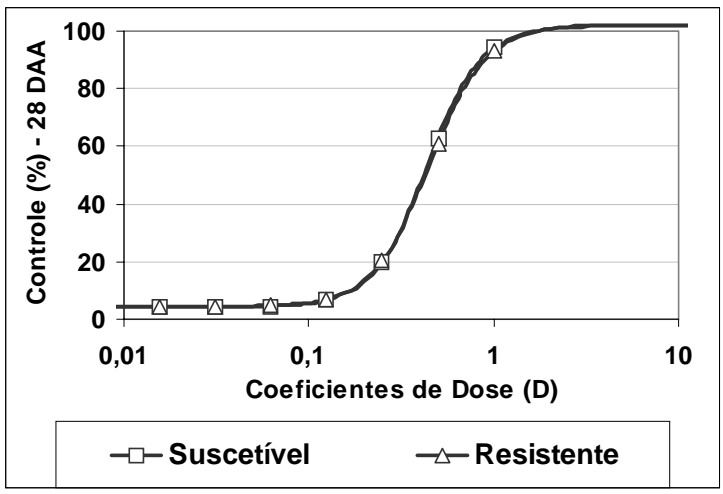

Figura 10 - Controle percentual de dois biótipos de capimcolchão, avaliado aos 28 DAA, quando submetidos à aplicação de iodosulfuron + foramsulfuron. Piracicaba, 2005.

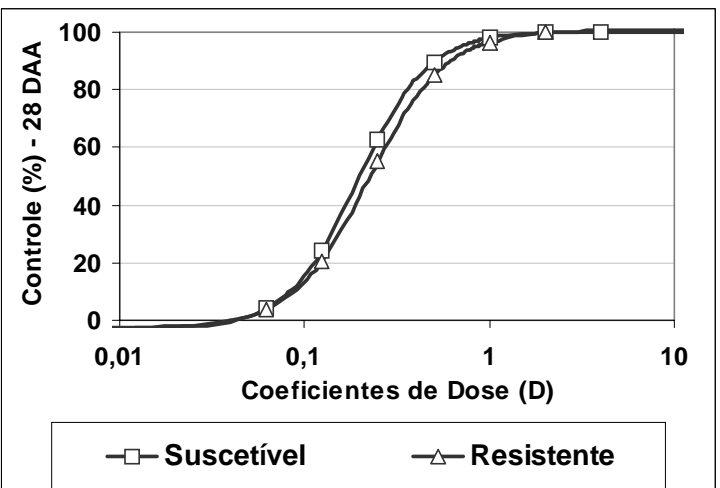

Figura 11 - Controle percentual de dois biótipos de capimcolchão, avaliado aos 28 DAA, quando submetidos à aplicação de mesotrione. Piracicaba, 2005.

Planta Daninha, Viçosa-MG, v. 24, n. 4, p. 789-796, 2006 
Nesse caso, em áreas com problemas de biótipos de capim-colchão resistentes aos inibidores da ACCase, há a possibilidade de uso do herbicida imazethapyr, na cultura da soja; e dos herbicidas nicosulfuron, iodosulfuron + foramsulfuron e mesotrione, na cultura de milho em sucessão. No entanto, no Brasil, os casos mais freqüentes de resistência de plantas daninhas a herbicidas estão relacionados com os inibidores da ALS, sobretudo na cultura da soja, embora, até o momento, não haja registros de resistência de capim-colchão a estes herbicidas. Todavia, não existem relatos de resistência para os inibidores da biossintese de carotenos (Christoffoleti \& López-Ovejero, 2004), de modo que se apresentam como opções bastante interessantes para o manejo da resistência do capim-colchão.

A partir dos resultados obtidos, conclui-se que: o biótipo $\mathrm{R}$ é realmente resistente aos herbicidas inibidores da ACCase com niveis variáveis de resistência cruzada aos herbicidas dos grupos químicos dos APP e CHD; os herbicidas haloxyfop-r, propaquizafop e tepraloxydim, nas doses recomendadas, controlaram o biótipo $\mathrm{R}$ de forma satisfatória e semelhante à do biótipo S; e os biótipos R e S foram igualmente controlados pelos herbicidas imazethapyr, nicosulfuron, foramsulfuron + iodosulfuron e mesotrione, excluindo a hipótese de resistência múltipla no biótipo R de capim-colchão.

\section{LITERATURA CITADA}

BOERBOOM, C. M. Nonchemical options for delaying weed resistance to herbicides in Midwest cropping systems. Weed Technol., v. 13, p. 636-642, 1999.

CARVALHO, J. C. Mecanismo de ação dos herbicidas e sua relação com a resistência a herbicidas. In: CHRISTOFFOLETI, P. J. (Coord.). Aspectos da resistência de plantas daninhas a herbicidas. Londrina: Associação Brasileira de Ação a Resistência de Plantas aos Herbicidas, 2004. p. 23-48.

CARVALHO, S. J. P. et al. Curvas de dose-resposta para avaliação do controle de fluxos de emergência de plantas daninhas pelo herbicida imazapic. Planta Daninha, v. 23, n. 3, p. 535-542, 2005.

CHRISTOFFOLETI, P. J. Curvas de dose-resposta de biótipos resistente e suscetível de Bidens pilosa $\mathrm{L}$. aos herbicidas inibidores da ALS. Scientia Agrícola, v. 59, n. 3, p. 513-519, 2002.
CHRISTOFFOLETI, P. J.; LÓPEZ-OVEJERO, R. F

Definições e situação da resistência de plantas daninhas aos herbicidas no Brasil e no mundo. In: CHRISTOFFOLETI, P. J. (Coord.). Aspectos de resistência de plantas daninhas a herbicidas. 2.ed. Campinas: Associação Brasileira de Ação a Resistência de Plantas aos Herbicidas, 2004. p. 3-22.

CHRISTOFFOLETI, P. J.; VICTORIA FILHO, R.; SILVA,

C. B. Resistência de plantas daninhas aos herbicidas. Planta Daninha, v. 12, n. 1, p. 13-20, 1994.

CHRISTOFFOLETI, P. J.; KEHDI, C. A.; CORTEZ, M. G. Manejo da planta daninha Brachiaria plantaginea aos herbicidas inibidores da ACCase. Planta Daninha, v. 19, n. 1, p. 61-66, 2001.

De PRADO, R.; OSUNA, M. D.; FISCHER, A. J. Resistance to ACCase inhibitor herbicides in a green foxtail (Setaria viridis) biotype in Europe. Weed Science, v. 52, p. 506-512, 2004.

GAZZIERO, D. L. P. et al. Resistência da planta daninha capim-marmelada (Brachiaria plantaginea) aos herbicidas inibidores da enzima ACCase na cultura da soja. Planta Daninha, v. 18, n. 1, p. 169-184, 2000.

GOULD, F. Comparisons between resistance management strategies for insects and weeds. Weed Technology, v. 9, p. 830-839, 1995.

GRESSEL, J.; SEGEL, L. A. Modeleing the effectiveness of herbicide rotation and mixture strategies to delay or preclude resistance. Weed Technology, v. 4, p. 186-198, 1990.

HALL, L. M.; STROME, K. M.; HORSMAN, G. P. Resistance to acetolactate synthase inhibitors and quinclorac in a biotype of false clover (Gallium spurium). Weed Science, v. 46, p. 390-396, 1998.

LÓPEZ-OVEJERO, R. F. et al. Resistência de populações de capim-colchão (Digitaria ciliaris) aos herbicidas inibidores da Acetil Co-A Carboxilase. Planta Daninha, v. 23, n. 3, p. 543-549, 2005.

MONQUEIRO, P. A.; CHRISTOFFOLETI, P. J.; DIAS, C. T. S. Resistência de plantas daninhas aos herbicidas inibidores da ALS na cultura da soja (Glycine max). Planta Daninha, v. 18 , n. 3, p. 419-425, 2000.

POWLES, S. B.; HOLTUM, J. A. M. Herbicide resistance in plants: biology and biochemistry. Boca Raton: Lewis, 1994. $353 \mathrm{p}$.

SEEFELDT, S. S.; JENSEN, J. E.; FUERST, E. P. Loglogistic analysis of herbicide dose-response relationship. Weed Technology, v. 9, p. 218-227, 1995.

VOLEMBERG, D.; STOLTENBERG, D. Altered acetylcoenzyme A carboxylase confers resistance to clethodim, fluazifop and sethoxydim in Setaria faberi and Digitaria sanguinalis. Weed Research, v. 42, p. 342-350, 2002. 